\title{
Génération de la commande des systèmes de production en ingénierie intégrée produit/processus
}

\author{
Jean-Marc Faure, Florent Couffin \\ ISMCM, 3 rue Fernand Hainaut, 93407 Saint-Ouen Cedex \\ E-mail : jean-marc.faure@ ismcm-cesti.fr, florent.couffin@ismcm-cesti.fr
}

\section{Résumé}

Cet article présente certains de nos travaux effectués en vue d'améliorer l'intégration des activités du cycle de vie des systèmes de production, c'est-à-dire la communication entre les différents acteurs de ce cycle, ainsi que l'intégration entre ces activités et celles relatives à la conception du produit et de son processus d'élaboration. La méthode retenue est l'intégration par les données à l'aide de modèles de référence, afin de faciliter la réutilisation des connaissances métier, et de méta-modèles. Deux applications développées en partenariat avec des industriels, à savoir un système d'aide à la maintenance et une méthode de génération systématique de la commande d'un processus, sont décrites.

\begin{abstract}
We present in this article our works performed in order to improve the integration of production systems life-cycle activities, as well as the integration of these activities with those related to product and manufacturing process design. Our method uses integration by means of data and is based on reference models and meta-models; this facilitates knowledge reuse. Two industrial applications : troubleshooting aid system development and systematic generation of a process control are described.
\end{abstract}

\section{$1 \quad$ Introduction}

La conception, la réalisation et l'exploitation des systèmes automatisés de production nécessitent l'exécution de nombreuses activités (spécification, conceptions générale et détaillée, tests, maintenance, ...) dont l'enchaînement peut être décrit par le classique modèle du cycle de vie en V [16], ou par d'autres modèles [17] montrant la place de l'homme dans le système de production. A chaque activité peuvent être associés un ou plusieurs outils supports d'activité (logiciels de XAO) offrant une assistance informatique aux acteurs réalisant cette activité. Certaines de ces activités sont reliées à des activités du cycle de vie du produit élaboré par le système de production (la conception de la partie physique d'une machine automatique requiert par exemple d'avoir des indications sur le produit et sa gamme de fabrication).

La complexité des systèmes de production croissant, la maîtrise de ces activités tout au long du cycle de vie s'avère de plus en plus difficile. Dans le cadre de l'entreprise étendue ou entreprise partagée [11] dans laquelle un partenariat à long terme entre l'entreprise (pivot) et ses sous-traitants (partenaires) est prôné, cette difficulté est amplifiée par le nombre d'acteurs et la diversité de leurs activités et de leurs cultures. L'amélioration de la productivité passe cependant par la coopération entre tous ces acteurs, ce qui nécessite l'intégration de leurs processus (ordonnancement cohérent des activités) et l'intégration des informations utilisées par les activités. Nous nous limitons dans cet article à ce dernier point.

Pour réaliser l'intégration des informations utilisées par les activités, deux approches sont envisageables (Fig. 1) :

- le couplage direct entre activités pour lequel il est nécessaire d'avoir une interface entre chaque activité ;

- l'intégration par les données dans laquelle les activités communiquent au moyen d'un système d'information conceptuellement unique et commun à toutes les activités.

Notre choix [3] s'est porté sur la solution privilégiant l'intégration par les données. L'intégration par les données réduit en effet le nombre d'interfaces, facilite le parallélisme des activités (donc des outils supports de ces activités) lors de la conception, et permet de réduire la phase d'industrialisation et d'obtenir des bases de données intégrées qui documentent les processus automatisés [5]. Ce mode d'intégration privilégie l'ingénierie simultanée en permettant aux activités ou outils supports d'activité d'accéder simultanément aux données les concernant. L'ingénierie simultanée instaure alors un partage de l'information entre le pivot et les partenaires basé sur la confiance et permet le transfert des innovations et des technologies d'un partenaire à un autre en mettant en œuvre une synergie entre des partenaires d'horizons différents [13]. 


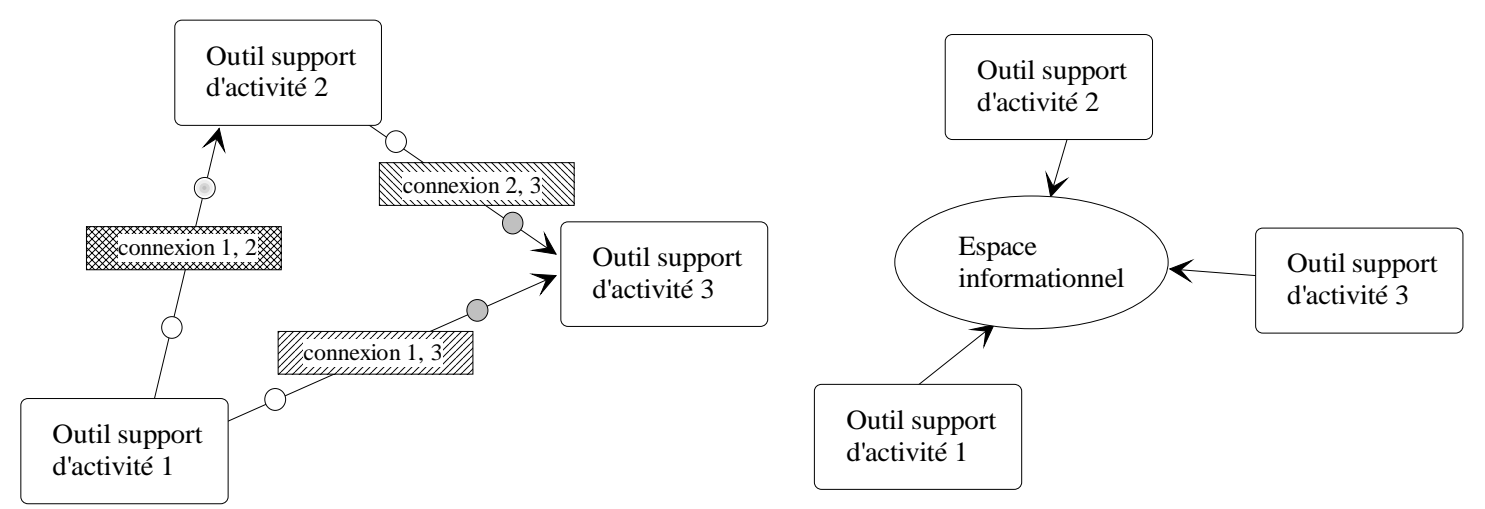

Figure 1 : Intégration par les activités et intégration par les données

La mise en pratique de l'intégration par les données nécessite en pratique la conception de bases de données partagées, et/ou la définition de standards d'échange entre logiciels d'assistance. Bien que l'espace informationnel soit conceptuellement unique, des contraintes opérationnelles peuvent en effet nécessiter la mise en œuvre de l'une ou de l'autre ou de ces deux types de solutions. Dans les deux cas, l'attention est plus particulièrement portée sur le système d'information fédérant les activités du cycle de vie du système de production.

Dans la suite de cet article, nous présenterons tout d'abord notre méthode de conception de ce système d'information pour l'intégration; cette méthode s'appuie sur deux concepts permettant la formalisation de la communication entre acteurs: modèles de référence pour la réutilisation des connaissances et méta-modèles pour la formalisation des langages de représentation. Nous montrerons ensuite comment plusieurs normes relatives à l'ingénierie des systèmes de production considèrent l'approche d'intégration par les données ainsi que le concept de modèle de référence. Nous décrirons enfin deux applications développées en utilisant la méthode précédemment définie.

\section{Principe et concepts pour l'ingénierie intégrée ${ }^{1}$}

Les activités concernées par l'intégration peuvent appartenir à la même phase du cycle de vie ; on parle alors d'intégration synchronique. Lorsque les activités qui échangent ou partagent des données sont positionnées dans des phases différentes du cycle de vie, l'objectif poursuivi sera leur intégration diachronique (au cours du temps). A titre d'exemple, l'intégration des phases de conception et de fabrication d'une pièce mécanique (problème de CFAO Mécanique) est un cas d'application d'intégration diachronique. La communication entre les concepteurs des schémas électriques d'un système automatisé et les concepteurs des programmes d'automates est un exemple d'intégration synchronique.

Ces qualificatifs ont été proposés pour la première fois par Ferdinand de Saussure, dans son Cours de Linguistique générale, publié en 1916. Selon Saussure, la synchronie désigne l'état dans lequel se trouve une langue à un moment déterminé de son histoire ; la diachronie désigne l'évolution de la langue. Cette utilisation de termes employés originellement en Linguistique n'est pas le fruit du hasard, mais correspond à une volonté de privilégier l'univers du discours des acteurs du cycle de vie comme facteur primordial de l'intégration.

Nous pensons en effet que la réussite de l'intégration par les données d'activités nécessite la définition précise de l'univers du discours des acteurs réalisant ces activités. Ceci constitue le principe de base de notre méthode.

Pour représenter précisément un univers du discours, plusieurs formalismes [6], [10], [15] sont envisageables (NIAM, z, entité-relation, EXPRESS-G, ...); nous ne détaillerons pas ici les avantages et inconvénients de chacun de ces formalismes. On obtient en final un modèle dénommé :

- modèle de référence [9], modèle conceptuel de données générique exprimant en termes de connaissances métier l'univers du discours d'un groupe d'acteurs du cycle de vie et permettant la réutilisation des connaissances. Ce modèle permet la conception du système d'information pour l'intégration au moyen d'un processus de spécialisation comme nous le décrirons au paragraphe 4.1.

- méta-modèle lorsque l'univers du discours considéré est celui d'un langage de représentation (langage d'analyse fonctionnelle, grafcet, ...) permettant de construire des modèles descriptifs de certains vues du

\footnotetext{
${ }^{1}$ Nous préférons cette traduction du terme anglais concurrent engineering à celle, trop directe selon nous et peu explicite, d'ingénierie concourante.
} 
système. Un méta-modèle est une représentation des concepts et des règles du langage de représentation et permet de réaliser et d'intégrer des environnements de conception de modèles (éditeurs spécialisés).

Nous considérons que le développement de ces deux types de modèles est un prérequis indispensable à une intégration réussie. Bon nombre de cas d'échecs que nous avons pu rencontrer étaient dus en effet en une incompréhension mutuelle des acteurs devant communiquer. L’ingénierie intégrée requiert une mise en commun de connaissances métier et l'emploi de langages de représentation dépourvus d'ambiguité.

\section{La normalisation en ingénierie des systèmes de production}

Plusieurs projets de recherche en collaboration avec l'industrie ont eu pour objectif le développement de normes pour l'ingénierie intégrée [17]. Sans souci d'exhaustivité, nous souhaitons à présent montrer comment l'approche d'intégration par les données ainsi que le concept de modèle de référence sont pris en compte dans certaines normes ou projets de normes. De par son caractère générique, un modèle de référence a en effet pour vocation d'être normalisé ; notre volonté de bâtir le système d'information pour l'intégration à partir de modèles privilégiant l'univers du discours nous impose donc de nous intéresser à la normalisation dans notre domaine d'étude.

\subsection{Le cadre de modélisation CIMOSA}

Ce modèle [2] développé par le consortium AMICE comporte deux parties :

- un cadre de modélisation permettant la création de modèles correspondant aux différentes phases du processus de développement (spécification, conception, réalisation), selon différents points de vue (vues fonction, information, organisation, ressource), et à trois niveaux de généricité (générique, partiel et particulier) ;

- un modèle de la structure d'accueil informatique nécessaire à l'intégration de ces modèles : IIS (Integrating InfraStructure).

CIMOSA est un modèle retenu par le Comité Européen de Normalisation (CEN) en vue du développement de normes pour la modélisation d'entreprise. Les travaux du groupe de travail 1 de la commission technique 310 (CEN /TC 310/WG1) ont abouti aux résultats suivants :

- pré-norme ENV 40003 Framework for Enterprise Modelling, sur la structure du cadre de modélisation, depuis avril 1990 ;

- pré-norme ENV 12204 Constructs for Enterprise Modelling, sur les primitives de modélisation, depuis 1995 ;

- travaux préparatoires à une pré-norme sur la structure d'accueil informatique, baptisée EMEIS (Enterprise Model Execution and Integration Services).

Le concept de modèle de référence (appelé modèle partiel) est présent dans le cadre de modélisation CIMOSA, en tant que niveau intermédiaire sur l'axe de généricité. On peut simplement regretter que les règles de passage d'un modèle partiel à un modèle particulier ne soient pas indiquées ; cette constatation nous a amené à développer les recherches qui ont conduit au processus de spécialisation d'un modèle de référence exprimé sous forme de modèle conceptuel de données présenté au paragraphe 4.1 .

\subsection{La norme BASE-PTA}

Cette norme [1] a été élaborée à l'initiative du Centre Coopératif de Génie Automatique (CCGA), regroupant des fournisseurs de constituants d'automatisme, des utilisateurs et des laboratoires universitaires. Elle décrit un modèle de référence des données consommées ou produites par les activités relatives au système de contrôle-commande d'un système de production.

Ce modèle comporte quatre parties (Fig. 2). La première permet de décrire la structure du système de contrôlecommande. Deux autres parties décrivent les composants logiciels et matériels de ce système ; la réunion de ces deux parties est un modèle de la fourniture de l'automaticien. Enfin une dernière partie permet de faire le lien avec d'autres données, comme celles contenues dans des manuels de configuration, de programmation. Bien évidemment ces quatre parties ne sont pas séparées ; il existe des relations entre les classes d'entité de chacune d'elles. 
Il convient de noter que la partie spécification/structuration permet d'établir des liens avec des modèles de référence caractéristiques de la gamme de fabrication, assurant ainsi une intégration possible entre activités de conception du processus physique et activités de conception de la commande de ce processus.

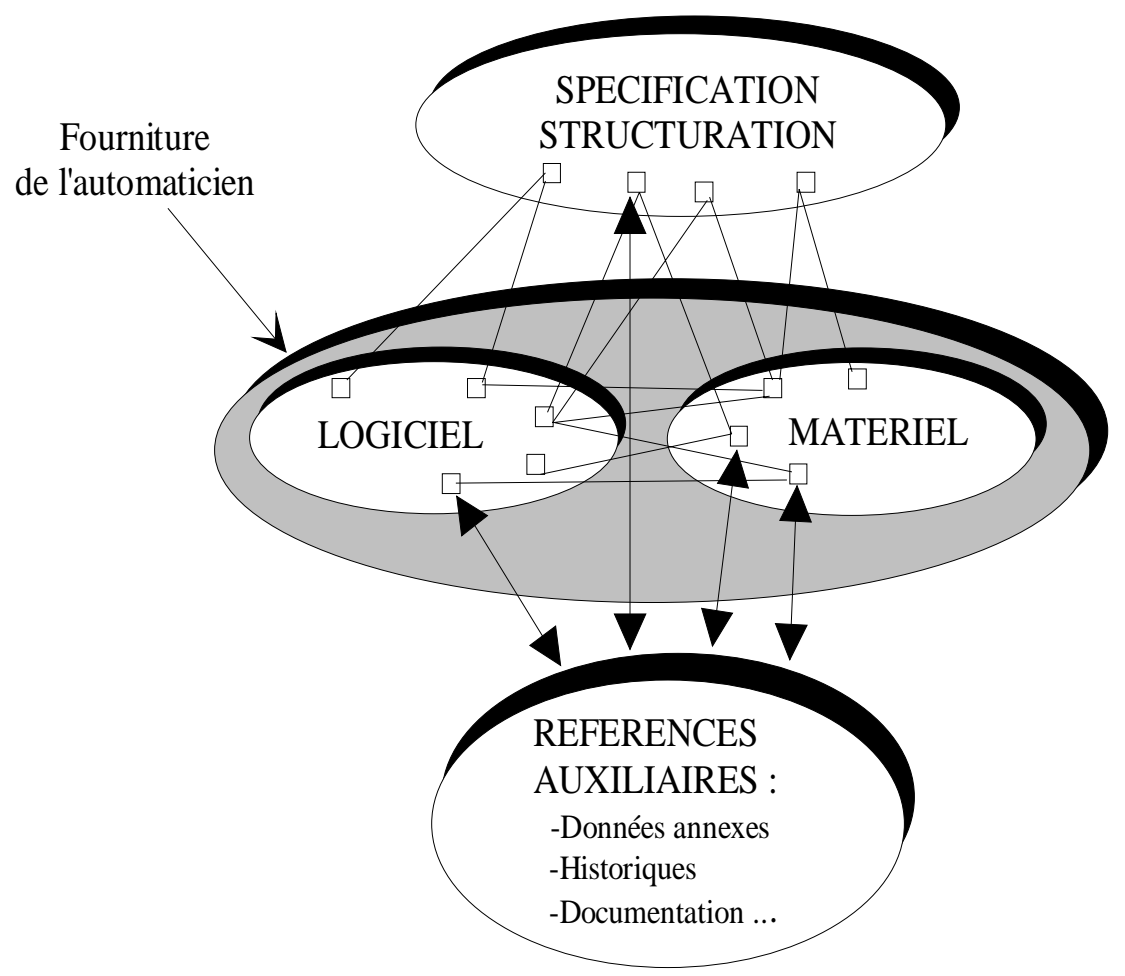

Figure 2 : Une vision synthétique de la structure de BASE-PTA

La norme BASE-PTA reprend le principe d'intégration par les données et propose de construire le système d'information à partir du modèle de référence par un processus de spécialisation. Nous pensons cependant que le document normatif actuel ne permet pas de développer facilement une application, le processus de spécialisation y étant trop sommairement décrit et aucun exemple n'y figurant. Nous espérons que les travaux réalisés, tels que ceux présentés ci-après, permettront de pallier ces inconvénients.

\section{$4 \quad$ Intégration d'activités à l'aide d'un modèle de référence}

Nous souhaitons à présent montrer comment il est possible, à partir d'un modèle de référence exprimé sous forme de modèle conceptuel de données, d'élaborer le système d'information pour l'intégration. Nous décrivons d'abord succinctement le processus de spécialisation qui permet d'obtenir un modèle particulier, caractéristique des activités et des outils logiciels à intégrer. Puis nous présentons une application concrète mettant en jeu le modèle de référence de la norme BASE-PTA et ce processus.

\subsection{Processus de spécialisation d'un modèle de référence}

Ce processus (Fig. 3) permet la construction d'un modèle de données appliqué à partir d'un modèle de données de référence ; il s'apparente à l'instanciation d'un modèle partiel en un modèle particulier de la vue information de CIMOSA. Pour obtenir le modèle particulier, que nous avons dénommé SSCA (sous-schéma conceptuel appliqué), la première phase du processus consiste à extraire du modèle de référence un ensemble connexe de classes d'entité et de relation, qualifié de SSC (sous-schéma conceptuel), caractéristique des activités concernées (par exemple les activités de conception des schémas électriques). A partir de ce SSC, on obtient le SSCA en particularisant les classes en fonction des activités spécifiques concernées (par exemple conception des schémas de câblage). 


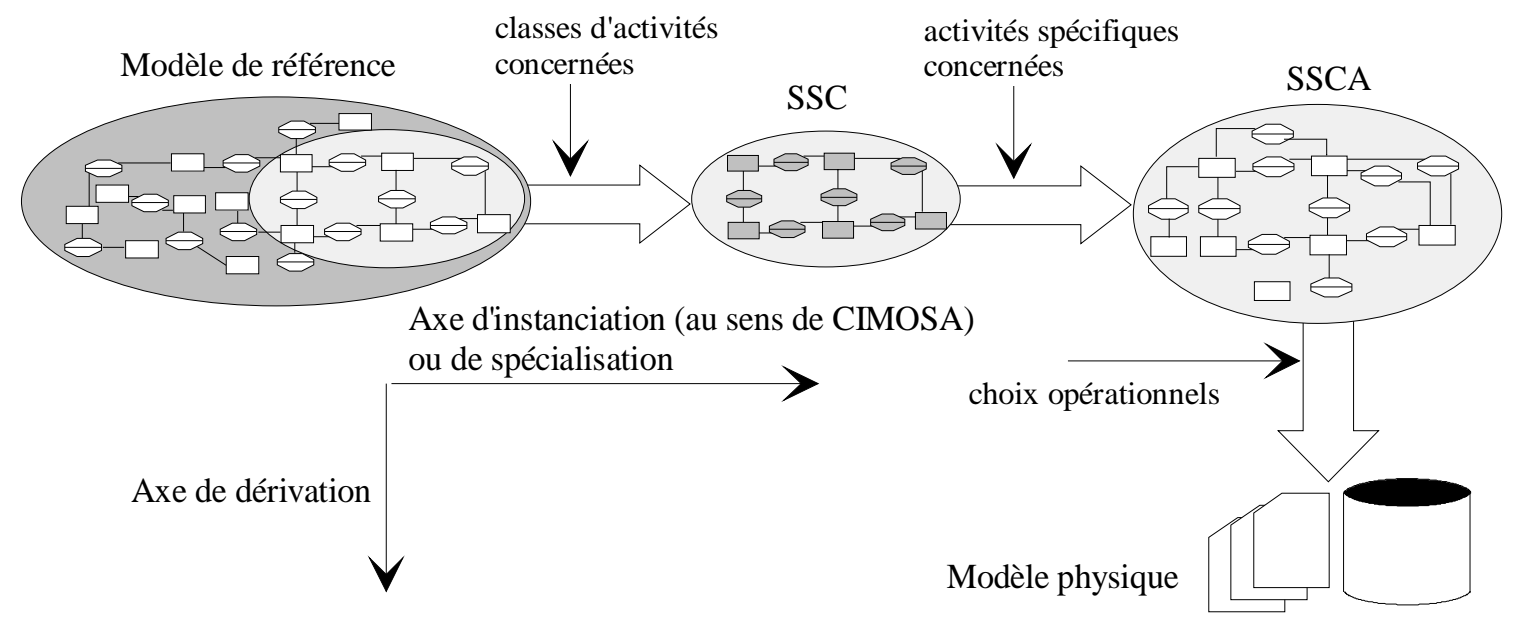

Figure 3 : Du modèle de référence au modèle particulier

Le processus permettant de construire un SSCA résultat à partir d'un modèle de référence comporte quatre étapes utilisant quatre opérateurs, dont la formalisation fait appel à des prédicats sur les classes d'entité ou de relation [3]. Ces opérateurs permettent respectivement de sélectionner des classes d'entité et de relation, de spécialiser les classes d'entité sélectionnées, de spécialiser les classes de relation sélectionnées, et enfin de spécialiser certaines structures (rajout de contraintes entre classes d'objets). Cette approche assure la traçabilité du processus de conception du SSCA final.

Nous tenons à souligner l'importance de ce processus qui permet une réelle utilisation du concept de modèle de référence et qui, dans notre cas, nous a permis de déduire plusieurs modèles particuliers dont certains sont présentés au paragraphe suivant.

\subsection{Conception de l'aide à la documentation en maintenance}

Lors du dépannage d'un équipement automatisé de production, les opérateurs de maintenance doivent consulter un grand nombre de documents, contenus dans les dossiers électrique et logiciel de l'équipement. Ces dossiers contiennent respectivement les schémas de principe et les schémas de câblage, ainsi que les modèles de conception du logiciel et les programmes d'automates.

Cette tâche de consultation de documents afin de localiser l'origine de la panne est coûteuse en temps. Aussi, dans un souci d'amélioration de la productivité des équipements de production par réduction des temps de maintenance corrective, nous avons entrepris, en collaboration avec la Direction des Techniques de Production et la Direction de la Recherche de Automobiles Renault, de concevoir une base de données d'aide à la documentation électronique en maintenance. Ce travail a été conduit dans le cadre du projet ESPRIT VOICE.

Nous tenons à souligner que l'objectif de cette base n'est pas simplement de stocker des dossiers sous forme électronique, mais qu'elle permet la navigation entre documents ; à titre d'exemple, les relations entre les borniers d'automates et les variables d'entrée-sortie des programmes peuvent être obtenues.

Une analyse fonctionnelle des activités de dépannage à l'aide de l'outil de modélisation de la vue fonction de CIMOSA a été tout d'abord conduite. Nous nous sommes intéressés ensuite à la conception de la structure de données de la base et à son alimentation, la quantité de données à stocker étant très importante. Ce dernier point induisait forcément une réflexion sur les fichiers d'échange entre les logiciels supports d'activités de conception, XELEC pour la schématique électrique et EDIDOS pour le logiciel, et le logiciel d'aide à la documentation en dépannage.

Pour définir la structure de la base et de ces fichiers d'échange (Fig. 4), nous avons utilisé le modèle de référence BASE-PTA, qui contient des classes d'entité caractéristiques de la fourniture de l'automaticien, et le processus de spécialisation précédemment décrit, qui a permis d'obtenir les schémas appliqués.

Cette recherche constitue selon nous un bon exemple d'intégration diachronique entre la phase de conception et la phase de maintenance. Le système d'aide au dépannage a été testé auprès des utilisateurs qui l'ont considéré comme étant une évolution importante facilitant leurs opérations ; la critique principale faite concernait l'absence de liens avec la visualisation dynamique des programmes, fonction importante dans l'analyse de l'origine d'une panne à partir des états d'une variable automate. Pour donner quelques indications sur les caractéristiques de la base de données, nous indiquerons qu'une cellule d'assemblage tôlerie requiert 300 Mega-octets en moyenne, 70 étant nécessaires à la base et le reste servant au stockage des fichiers graphiques. 


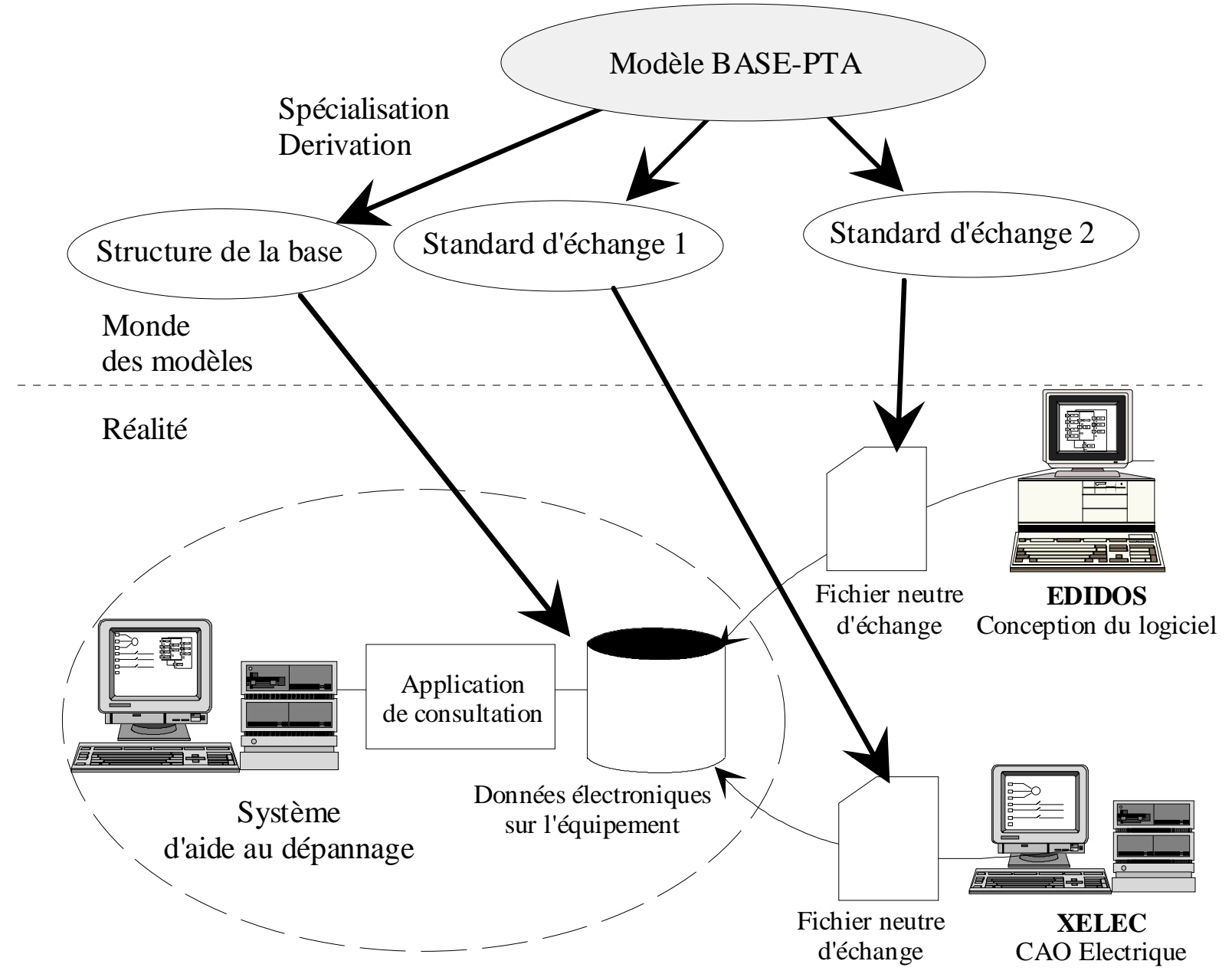

Figure 4 : Conception d'un système d'aide au dépannage

\section{Intégration à l'aide de méta-modèles : génération systématique de la commande d'un processus manufacturier}

Nous nous intéressons à présent à la conception de la commande d'un processus manufacturier, considéré comme un système à événements discrets. Cette conception requiert obligatoirement la connaissance du processus, constitué d'opérations ordonnées et exécutées par plusieurs ressources (robots, convoyeurs, opérateurs, ...). En adoptant le principe d'intégration par les données, il paraît très intéressant d'utiliser les résultats de la phase de conception du processus pour élaborer le modèle de sa commande.

En partenariat avec des industriels, nous avons donc développé une méthode permettant de construire un modèle statechart décrivant la commande d'un processus à partir de la donnée des éléments de ce processus. Nous avons retenu le formalisme des state-charts [7] pour décrire la commande car il offre de bonnes possibilités d'expression de la hiérarchie d'états, du parallélisme et de la communication. Il n'en demeure pas moins vrai que l'élaboration d'un modèle de commande décrit dans ce formalisme bénéficie grandement d'une aide méthodologique permettant de structurer le modèle.

Après avoir exposé notre méthode et indiqué comment nous avons pu l'automatiser en partie, nous montrerons son application dans le cas de la commande d'une cellule d'assemblage d'éléments de carrosserie automobile.

\subsection{Méthode de génération du modèle de commande}

La méthode que nous avons développée est schématisée par la figure 5. Elle comporte trois étapes dont l'une (en grisé sur la figure) est automatique, les deux autres nécessitant l'intervention des concepteurs, et est basée sur deux métamodèles : 
- méta-modèle de processus

- méta-modèle des state-charts.

Ces méta-modèles, que nous détaillons par la suite, représentent respectivement les univers du discours (ensemble des concepts du domaine et des relations entre ces concepts) permettant de décrire un processus ou un modèle state-chart. Ils ont tous deux été exprimés à l'aide des formalismes OMT, afin de bénéficier du pouvoir d'expression et de la large diffusion de cet outil de modélisation, et EXPRESS, afin de pouvoir en déduire des standards d'échange entre logiciels de CAO. La mise en correspondance de ces deux méta-modèles a permis de définir des règles de traduction automatique permettant la construction d'un modèle state-chart en connaissant l'ordonnancement des opérations au sein du processus.

Cette méthode a donné lieu au développement d'un logiciel qui assiste le concepteur lors des étapes " Elaboration du processus" et "Enrichissement du modèle de commande" et qui génère automatiquement le modèle de base de la commande.

Nous souhaitons à présent indiquer la structure des deux méta-modèles et montrer leur intérêt pour la génération du modèle de commande.

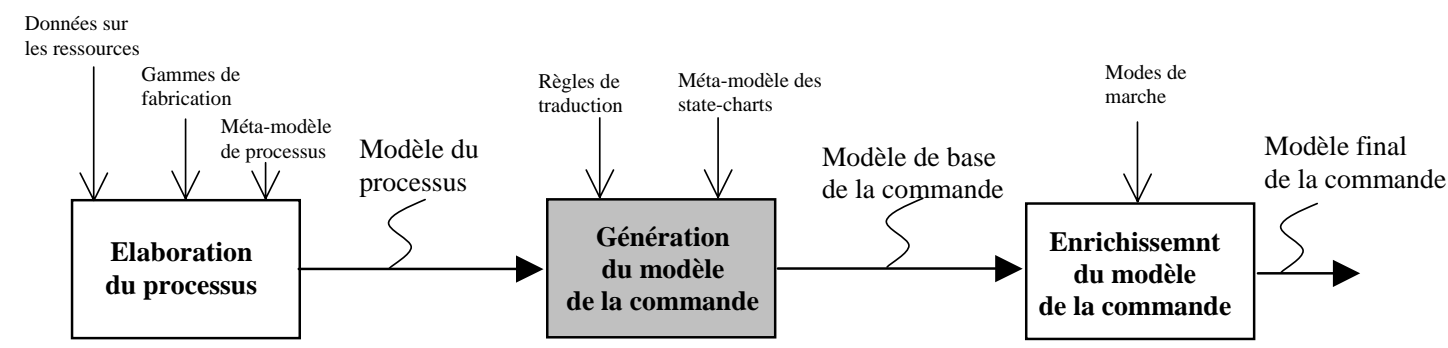

Figure 5 : Méthode de génération de la commande

\subsubsection{Méta-modèle de processus}

Issu du projet européen ESPRIT III SCOPES (Systematic COncurrent design of Products, Equipment and control Systems), ce méta-modèle est un schéma conceptuel de données qui contient les classes d'entité caractéristiques d'un processus ainsi que les classes de relation entre ces entités. Les classes d'entité principales pour notre étude sont l'ACTIVITE PHYSIQUE, élément du processus physique, l'ACTIVITE LOGIQUE, permettant d'ordonner les activités physiques, la RESSOURCE, et le FLOT_DE_CONTROLE, qui permet d'exprimer les communications entre les activités physiques. D'autres classes d'entité, telles que PIECE, COMPOSANT, CARACTERISTIQUE ne servent pas à générer le modèle de commande mais assurent la liaison avec le modèle du produit.

Connaissant la gamme de fabrication d'un produit et les ressources utilisables pour la réaliser, il est alors possible de construire un modèle du processus de fabrication de ce produit à l'aide de ces ressources par instanciation des éléments du méta-modèle. Ceci est effectué lors de la première étape de notre méthode.

\subsubsection{Méta-modèle des state-charts}

Nous avons élaboré ce méta-modèle en nous basant sur [8] qui précise utilement la sémantique du langage state-chart et sur des travaux antérieurs similaires relatifs au langage grafcet [4], [12]. Notons tout d'abord que, le langage state-chart étant un langage de description du comportement, son méta-modèle doit décrire ses aspects statique et dynamique. Notre méthode de génération du modèle state-chart de la commande ne concernant que la structure du modèle, nous nous limiterons à la description de la partie statique du méta-modèle.

Le méta-modèle obtenu rassemble tous les concepts du langage : état et super-état, transition, déclencheur, action, ... et précise les relations entre ces concepts. La figure 6 en présente un extrait, décrit en OMT. 


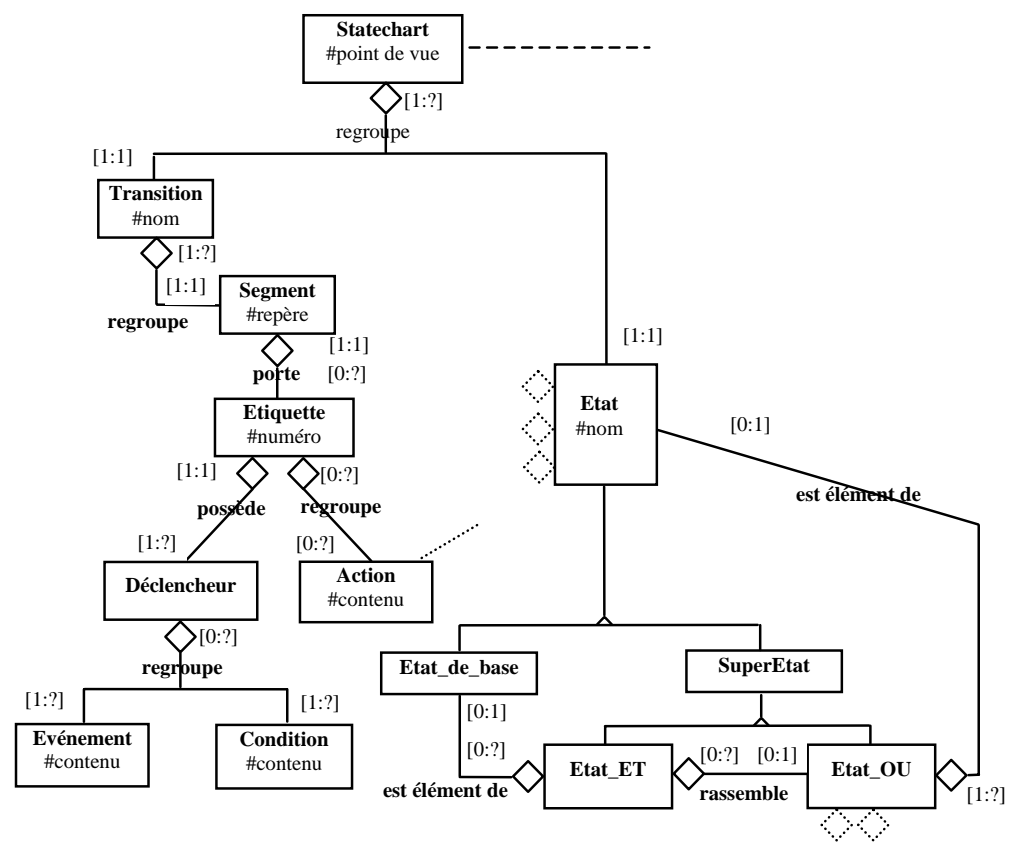

Figure 6 : Extrait du méta-modèle des state-charts

\subsubsection{Règles de traduction}

Connaissant le modèle du processus, ces règles permettent d'élaborer un state-chart décrivant sa commande. Elles ont été obtenues en cherchant à faire correspondre certaines classes du méta-modèle de processus aux classes du métamodèle des state-charts.

Nous ne détaillerons pas ici ces règles, décrites dans [14], mais indiquerons cependant quelques correspondances qu'elles utilisent :

- les ACTIVITES PHYSIQUES sont transformées en super-états OU ou en états de base, selon qu'elles sont ou non décomposables en d'autres activités ;

- les ACTIVITES LOGIQUES donnent naissance à des connecteurs OU ou à des super-états ET, selon leur type ;

- les FLOTS_DE_CONTROLE sont transformés en transitions.

La génération du modèle state-chart de la commande fait appel à un algorithme de parcours du modèle de processus qui élabore le modèle de commande en suivant ces règles. Cet algorithme a été implanté dans un logiciel qui réalise la deuxième étape de la figure 5 .

\subsubsection{Prise en compte des modes de marche}

Le modèle state-chart généré automatiquement est incomplet (c'est la raison pour laquelle il est qualifié de modèle de base dans la figure 5). Il ne comporte en effet aucune indication des modes de marche des ressources souhaités par l'utilisateur, tels que les procédures de démarrage et d'arrêt, les modes de fonctionnement dégradés, le traitement des défaillances des ressources, les reprises après défaillances.

Ces modes de marche ne sont en effet pas contenus dans le modèle de processus, qui ne considère que le fonctionnement normal du système de production, et il est donc obligatoire de compléter le modèle de base de la commande à l'aide éléments exprimant les modes de marche. Dans l'état actuel de nos travaux, cet enrichissement du modèle state-chart de base est réalisé par le concepteur en fonction des besoins exprimés dans le cahier des charges du système de production, par exemple sous la forme d'un GEMMA (Guide d'Etude des Modes de Marche et d'Arrêt). 


\subsection{Exemple d'application de la méthode}

Afin d'évaluer l'intérêt de la méthode et du logiciel de génération de la commande développés, nous avons traité plusieurs exemples industriels. Nous présentons ci-après un exemple issu de l'industrie automobile. Il s'agit d'un processus d'assemblage d'éléments de carrosserie. La réalisation de ce processus nécessite l'utilisation d'une cellule, schématisée à la figure 7, composée de cinq ressources : un opérateur, trois robots et une table tournante desservant les quatre autres ressources. La station 1 comporte un robot qui peut charger sur la table tournante une pièce de carrosserie et décharger le produit assemblé. A la station 2 un opérateur positionne trois accessoires sur la pièce brute ; deux de ces accessoires sont soudés à la pièce à la station 3 et le troisième l'est à la station 4.

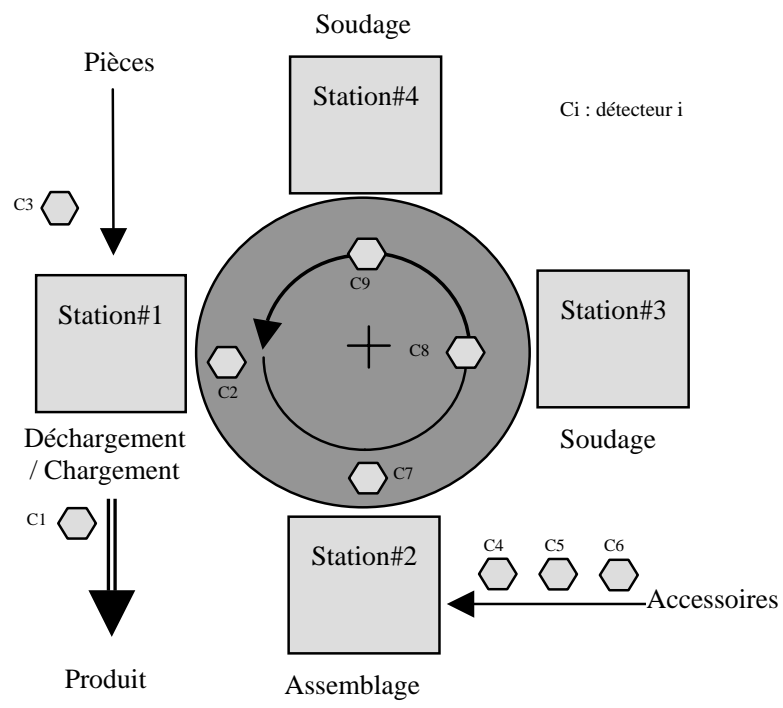

Figure 7 : Schéma de la cellule d'assemblage

L'application de la méthode à cet exemple permet d'obtenir le state-chart de la figure 8. Dans ce modèle seul le superétat PROCESSUS a été obtenu automatiquement, les états Arrêt Urgence et Repos, ainsi que les transitions reliées à ces états, ayant été rajoutés pour tenir compte des spécifications de modes de marche. La structure de ce super-état est calquée sur celle de la cellule.

Nous tenons d'autre part à préciser qu'à l'intérieur du super-état PROCESSUS, la transition entre les états Assemblage et Obtention ainsi que celle entre l'état Rotation et le super-état ASSEMBLAGE ont été rajoutées par le concepteur. Le modèle de processus ne s'intéresse en effet qu'aux activités physiques sur une seule pièce alors que notre modèle de commande cherche à tirer profit des possibilités de parallélisme offertes par l'organisation de la cellule

Cet exemple montre bien, nous le pensons, l'intérêt de la méthode développée ainsi que ses contraintes d'utilisation.

\subsection{Conclusion et perspectives}

La méthode de génération systématique de la commande d'un processus manufacturier que nous avons développée fournit une assistance réelle au concepteur automaticien. Elle se positionne dans un contexte d'ingénierie concourante produit-processus, et facilite donc l'intégration des activités de conception du produit, de conception du système physique de production et de conception de la commande de ce système.

Il est évident que les remarques faites précédemment et concernant l'ajout des reprises de séquences d'activités et des contraintes de modes de marche doivent être prises en compte par l'utilisateur de cette méthode.

A titre de perspectives, nous pensons que le méta-modèle des state-charts élaboré peut permettre le développement de techniques de vérification et de validation de modèles state-charts. Ceci afin de mieux assister les concepteurs de modèles de commande décrits dans ce langage. 


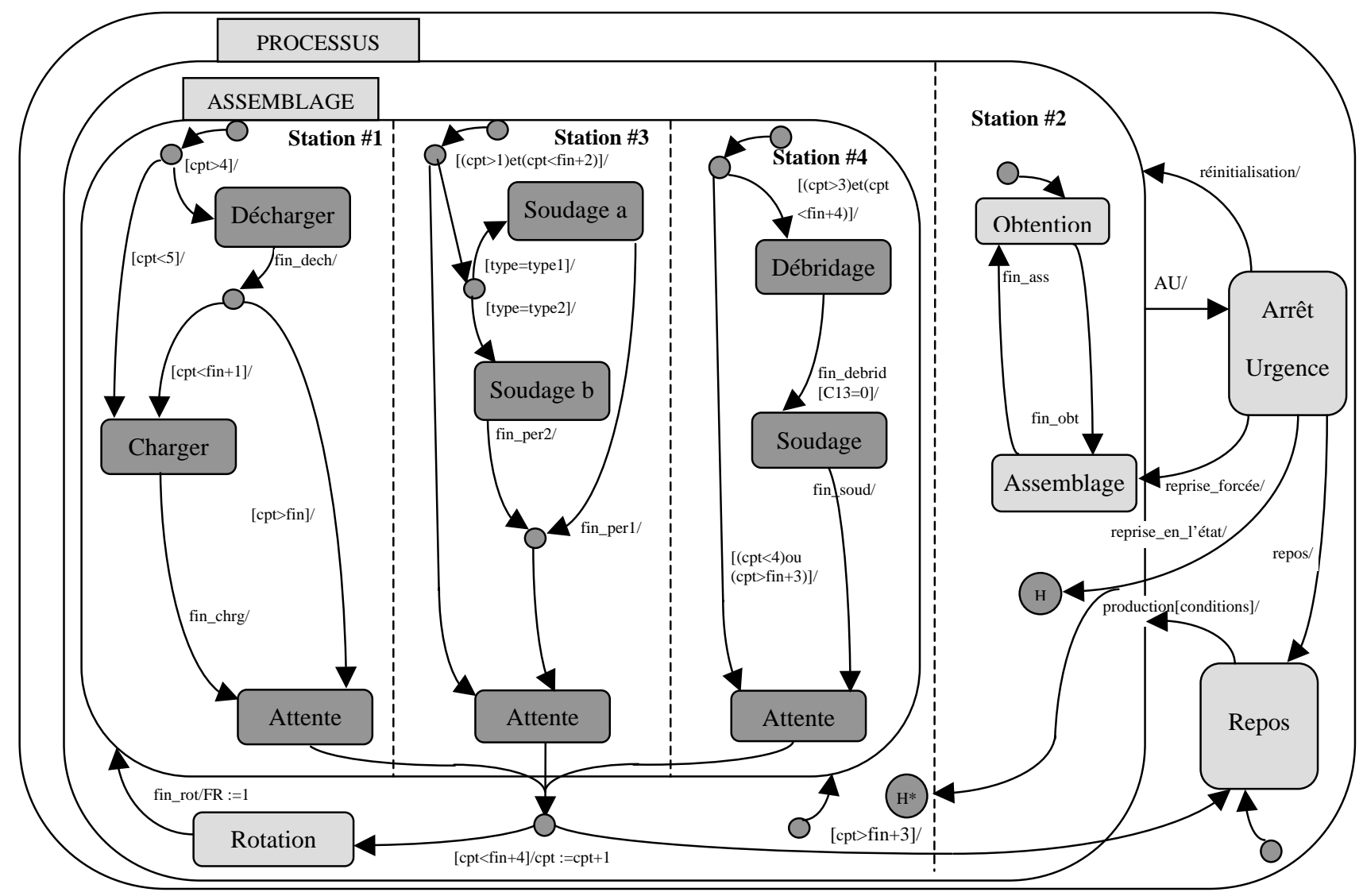

Figure 8 : Modèle state-chart final

\section{Conclusion}

La réduction des coûts et des délais de conception des systèmes de production passe par la mise en place de méthodes d'ingénierie intégrée (concurrent engineering en anglais). Les exemples présentés ont montré l'intérêt de l'approche d'intégration par les données et des concepts de modèle de référence et de méta-modèle pour faciliter cette évolution.

En particulier, l'intégration des activités de conception du processus de fabrication et de celles de conception de son système de commande doit permettre une amélioration importante dans la conception des automatismes.

Il n'en demeure pas moins vrai que la mise en place de ces méthodes nécessite une certaine ouverture des logiciels d'assistance et une volonté de compréhension d'autres cultures. S'il est relativement facile de trouver des solutions au second point, le premier dépend entièrement des politiques commerciales des industriels de l'offre. Il reste alors à souhaiter que la pression des utilisateurs pour des systèmes ouverts soit suffisante pour permettre cette nécessaire évolution des pratiques d'ingénierie. 


\section{Références}

[1] AFNOR, - Représentation des systèmes de contrôle et de commande des systèmes automatisés. Modèle BASEPTA, Norme AFNOR NF Z 68-901, 1996.

[2] AMICE ESPRIT Consortium, - CIMOSA : Open System Architecture for CIM, 2nd revised and extended version. Berlin, Allemagne. Editions Springer-Verlag. 1993.

[3] Couffin F., - Modèle de données de référence et processus de spécialisation pour l'intégration des activités de conception en génie automatique, Thèse de l'Ecole Normale Supérieure de Cachan, 14 janvier 1997.

[4] Couffin F., Lampérière S., Faure J.M., - Contribution to the Grafcet formalisation : A static meta-model proposition, Journal Européen des Systèmes Automatisés (JESA), Vol.31, n4/1997, pp 645-667.

[5] De Rooij N.J., - Integration of information : ILS in an international environment, Actes de la Troisième Conférence Internationale ILCE'95 : Concurrent Engineering \& Technical Information Processing, pp. 243252., Paris, France. 30 janvier - 3 février 1995.

[6] Habrias H., - Le modèle relationnel binaire : méthode IA (NIAM), Eyrolles, 1988.

[7] Harel D., - Statecharts : A visual formalism for complex systems, Science of Computer Programming, $\mathrm{n}^{\circ} 8, \mathrm{pp}$. 231-274, 1987.

[8] Harel D., Naamad A., - The STATEMATE Semantics of Statecharts, Wisdom Technical reports in Computer Science, CS95-31, 1995, revised in july 1996, ACM TOSEM, 1996.

[9] Hars A., Scheer W., - Reference model for entreprise wide data engineering, First international conference on entreprise integration modelling, pp. 320-328, Hilton Head, USA, 8-12 juin 1992.

[10] ISO - Product Data Representation and Exchange - Part 11 : The EXPRESS Language Reference Manual, ISO DIS 10303 - 11, août 1992.

[11] Julien P.A., - "L'entreprise partagée" : Contraintes et opportunités, Actes de la Troisième Conférence Internationale ILCE'95 : Concurrent Engineering \& Technical Information Processing, pp. 27-38. Paris, France. 30 janvier - 3 février 1995.

[12] Lhoste P., Faure J.M., Lesage J.J., Zaytoon J., - Comportement temporel du Grafcet, Journal Européen des Systèmes Automatisés (JESA), Vol.31, n4/1997, pp 695-711.

[13] Lombard-Gregori M., - Contribution au génie automatique : prototypage d'une architecture d'ingénierie concourante des systèmes intégrés de fabrication manufacturière, Thèse de doctorat de l'Université de Nancy I, février 1994.

[14] Monnereau C., - Génération de processus décrits par state-charts, Mémoire de recherche du DEA de Production Automatisée, Université Nancy I, septembre 1997.

[15] Morejon J., - MERISE : Vers une modélisation orientée objet, Les Editions d'Organisation, collection Ingénierie des systèmes d'information, 1994.

[16] Verdin L., - De la spécification à l'exploitation : le cycle de vie des Systèmes Automatisés de Production, Journées de synthèse "Génie Automatique et Production Industrielle", GAMI/ISMCM, Paris, 12-13 mars 1991.

[17] Williams T.J and al., - Architectures for integrating manufacturing activities and enterprises, Computers in Industry, vol. 24, n 2-3, pp. 111-139, 1994. 